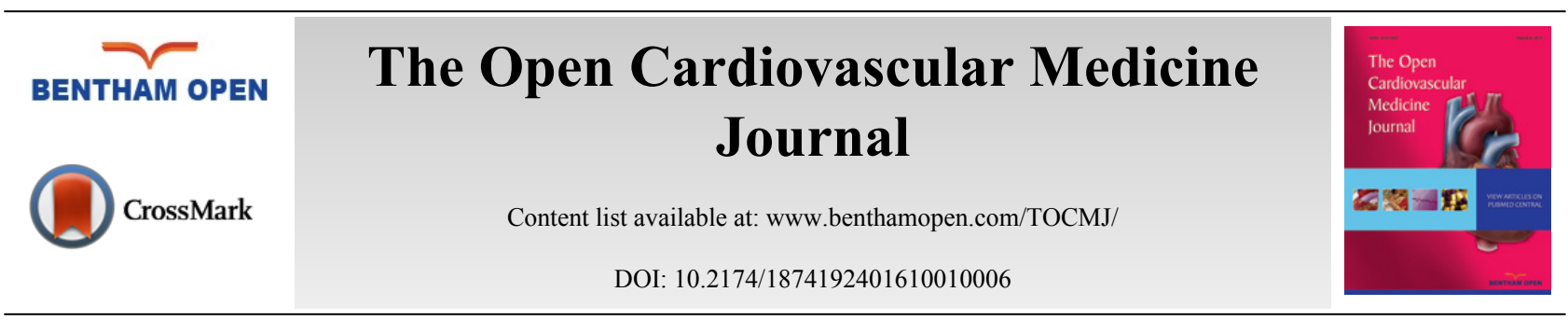

\title{
Pseudomonas aeruginosa Infective Endocarditis Following Transcatheter Aortic Valve Implantation: A Note of Caution
}

\author{
Juan Ignacio Dapás ${ }^{1}$, Cynthia Rivero ${ }^{1}$, Pablo Burgos ${ }^{2}$ and Andrea Vila ${ }^{1, *}$ \\ ${ }^{\prime}$ Department of Infectious Diseases, Hospital Italiano de Mendoza, Argentina \\ ${ }^{2}$ Department of Cardiovascular Surgery, Hospital Italiano de Mendoza, Argentina
}

\begin{abstract}
Transcatheter aortic valve implantation (TAVI) is an alternative treatment for severe aortic valve stenosis (AS) in patients with prohibitive risk for surgical aortic valve replacement (SAVR). Prosthetic valve endocarditis (PVE) is a rare complication of this relatively novel procedure and current guidelines do not include specific recommendations for its treatment.

We report a case of PVE due to Pseudomonas aeruginosa after TAVI that required SAVR, with successful outcome.

PVE usually occurs during the first year after TAVI and entails a high mortality risk because patients eligible for this minimally invasive procedure are fragile (i.e. advanced age and/or severe comorbidities). Additionally, clinical presentation may be atypical or subtle and transesophageal echocardiogram (TEE) may not be conclusive, which delays diagnosis and treatment worsening the prognosis. This case highlights that open SAVR might be ultimately indicated as part of treatment for TAVI-PVE despite a high-risk surgery score.
\end{abstract}

Keywords: Infective endocarditis, prosthetic valve endocarditis, Pseudomonas aeruginosa, TAVI, TAVI associated endocarditis, transcatheter aortic valve implantation.

\section{INTRODUCTION}

Valve disease is increasing as a consequence of population ageing. Up to $2 \%$ of patients older than 65 years have moderate or severe AS [1, 2]. SAVR dates back to 1960. Soon after its introduction it became the standard treatment for severe AS because it showed survival improvement [3]. Unfortunately, up to one-third of patients with severe AS are ineligible for SAVR because of advanced age or multiple comorbidities. Mortality risk associated with SAVR may be assessed using available algorithms. The most validated are the European System for Cardiac Operative Risk Evaluation (EuroSCORE) [4], and the Society of Thoracic Surgeons (STS) score [5]. Defining a high mortality risk for open surgery involves quantitative assessment (i.e., expected mortality $>20 \%$ with the EuroSCORE and $>10 \%$ with STS score) and qualitative multidisciplinary clinical judgment.

Since its first implementation in humans in 2002 [6], TAVI has increasingly been recognized as a minimally invasive therapeutic option for patients with severe symptomatic AS with high mortality risk for conventional SAVR. Success rates of TAVI are encouraging [7], thus a trend towards its use in a broader patient population may be expected. The two vascular approaches for TAVI are the retrograde transfemoral approach with balloon-expandable valve, and the anterograde transapical approach with self-expandable valve constructed of bovine pericardium on a metal stent. Transapical TAVI requires a small incision in the chest through an intercostal mini-thoracotomy but provides a stable platform for valve implantation due to the shorter distance for approaching the native valve. The apex of the heart is exposed, the prosthetic valve is compressed and placed on a catheter, which is passed through the apex and out across the patient's own aortic valve. A balloon is then used to expand the artificial valve into position.

\footnotetext{
* Address correspondence to this author at the Department of Infectious Diseases, Hospital Italiano de Mendoza, Avenida Acceso Este 1070, Guaymallén, Mendoza, Argentina; Tel: 542614608735 Email: santander@arlink.net.ar
} 
Despite being minimally invasive TAVI is associated with potentially serious complications. Although a multicentre trial showed similar 1-year survival rates between TAVI and SAVR in high-risk patients with severe AS [8], long-term outcomes have not been assessed.

The incidence of TAVI-PVE (up to 3\%) seems to be slightly higher to that of PVE following SAVR (1\%) [7, 9 11]. A recent large multicentre registry showed an incidence of TAVI-PVE of $1.1 \%$ (23 of 2,133) and $1.98 \%$ (6 of 303) after transfemoral and transapical approach, respectively [10]. In the same report the incidence of TAVI-PVE was higher within the first 12 months (80\%) suggesting a noso-comial source; in contrast to surgical PVE in which only $38 \%$ of cases occur within the first postoperative year [12]. Another clinical study showed that health care acquisition of the infection is more frequent in TAVI-PVE than in SAVR-PVE (71\% versus 36.5\%) [13].

In TAVI-PVE classic signs of endocarditis are infrequent and blood cultures are negative in up to $27 \%$ of patients [10].

As TAVI can be performed at the catheterization room there is concern about the safety of undertaking this procedure without high-efficiency particulate air (HEPA) filtered laminar airflow. A recent multicentre review showed that $55 \%$ of procedures were performed in the catheterization suite, $34 \%$ in a hybrid suite, and $11 \%$ in a surgical theatre [10]. One retrospective study found that performing TAVI in a catheterization laboratory was not associated with an increased risk of infective complications [14], as it has been described for implantable cardioverter-defibrillators and pacemakers [15]. Nevertheless this must need to be confirmed with prospective studies.

The management of TAVI-PVE has not been well established. There are no guidelines for indications and opportunity of surgical treatment, which is the most difficult decision in patients previously ineligible for open procedure due to a high mortality risk prediction.

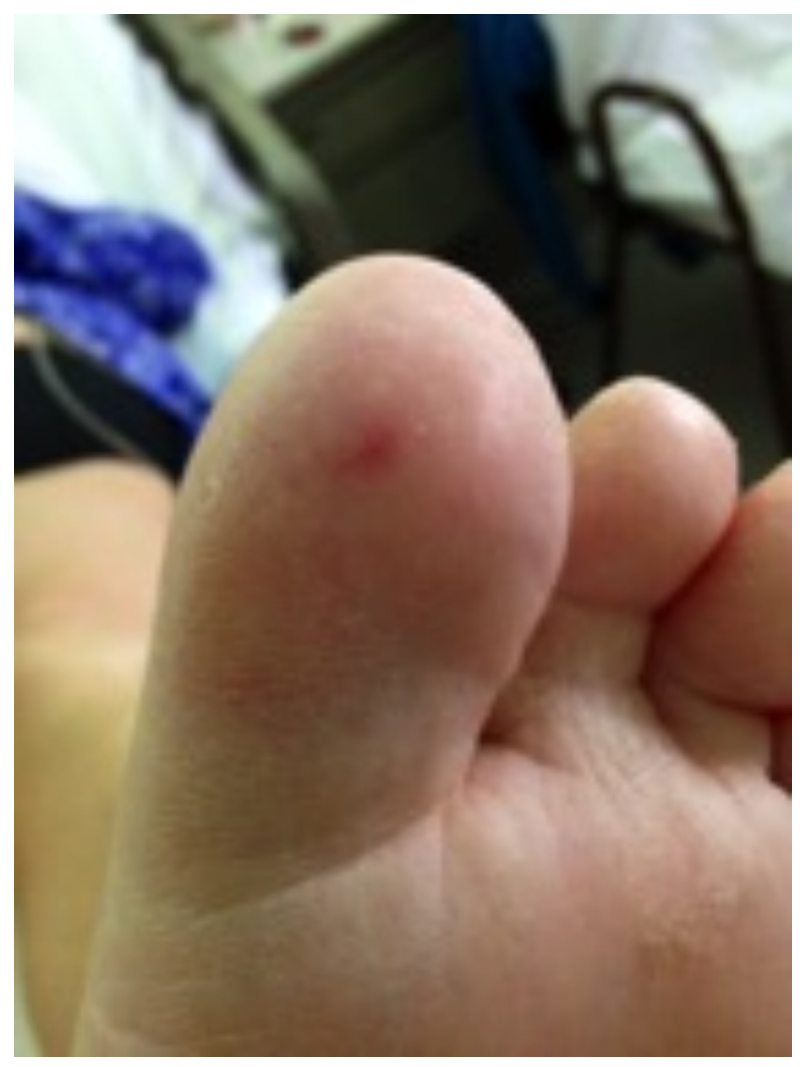

Fig. (1). Peripheral embolism.

\section{Case report}

A 62 years old female with medical history of arterial hypertension, atrial fibrillation, smoking, chronic obstructive pulmonary disease, obesity and bipolar affective disorder was admitted because of severe AS with heart failure. She had an expected mortality of $11.9 \%$ on logistic EuroSCORE and $20.5 \%$ on STS score. She refused open conventional surgery for valve replacement, thus she underwent transapical TAVI. The procedure was done in the catheterization 
room, which is not equipped with HEPA filter laminar flow. The intervention was done under strict sterile working conditions. Antibiotic prophylaxis consisted on piperacillin-tazobactam, vancomycin and amikacin.

One week later she presented with pleural empyema and bacteremia due to Pseudomonas aeruginosa (susceptible to ciprofloxacin, ceftazidime, cefepime, piperacillintazobactam, amikacin, colistin; and resistant to imipenem and meropenem). This complication was assumed as organ-space surgical site infection (SSI) and the patient was treated with pleural drainage and cefepime for 6 weeks with a favourable clinical outcome. At that time 2 transesophageal echocardiograms (TEE) (one at admission and the second 4 weeks later) were performed with no evidence of vegetation or perivalvular abscess.

Two weeks after completion of the 6-week antibiotic treatment she was readmitted with fever, a new aortic diastolic murmur and signs of peripheral embolism (Fig. 1). The patient was treated empirically with vancomycin and cefepime. A new TEE showed a periannular abscess (Movie (Supportive/Supplementary Material). P. aeruginosa with the same antibiotic resistance phenotype of previous isolation grew in all of 4 blood cultures. Laboratory tests were remarkable for a white blood cells count of 9,400 per $\mathrm{mm}^{3}$ (85\% neutrophils), haematocrit 34\%, haemoglobin $11.3 \mathrm{~g} / \mathrm{dL}$, erythrocyte sedimentation rate of $40 \mathrm{~mm} / \mathrm{hr}$ and C-reactive protein of $205 \mathrm{mg} / \mathrm{L}$. With diagnosis of early (<60 days) PVE due to $P$. aeruginosa complicated with periannular abscess an open SAVR was performed despite a logistic EuroSCORE of $78.5 \%$ and STS score of $54.9 \%$ at that time (Figs. 2-4). Drainage of the abscess and closure of the fistulous tract was performed with replacement of the aortic prosthesis with a homograft. Antimicrobial treatment was switched to piperacillin-tazobactam and amikacin for 2 weeks. She was discharged on ciprofloxacin and cefepime for completing additional 4 weeks as outpatient parenteral antibiotic therapy. After one year of follow up the patient is in good clinical condition with no evidence of infection relapse.

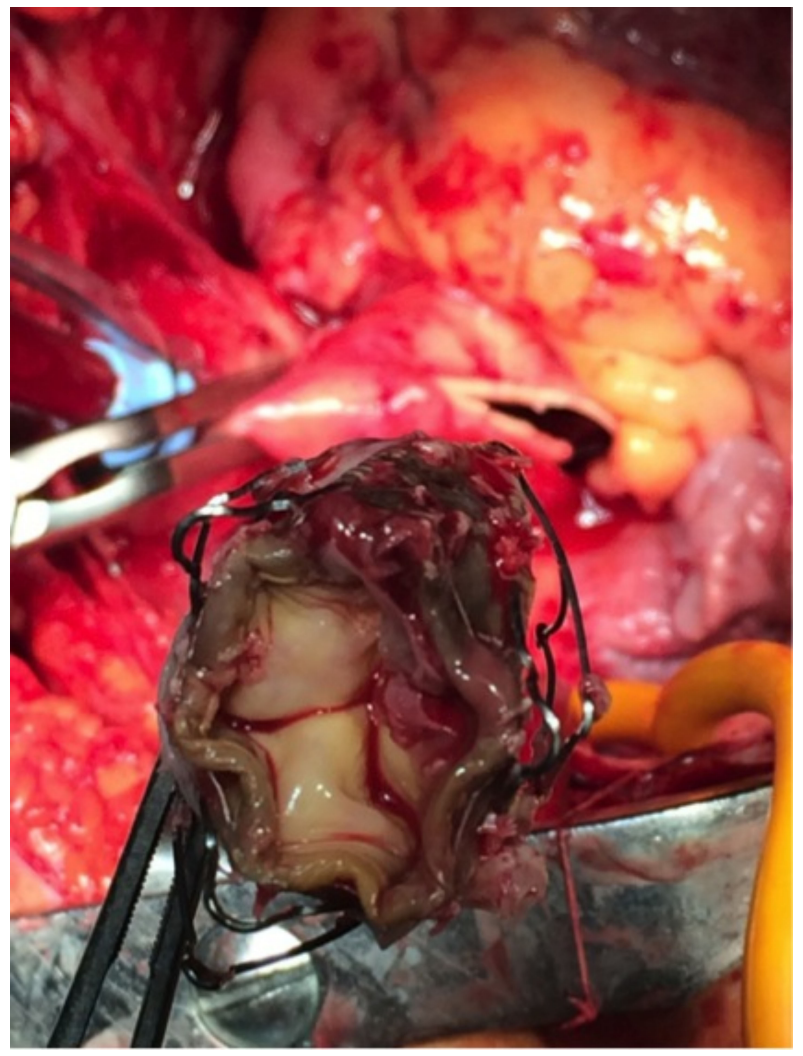

Fig. (2). TAVI explanted valve with vegetations.

\section{DISCUSSION}

AS is the most prevalent valve disease worldwide. TAVI is a novel procedure for high-risk patients suffering from severe AS. Despite being minimally invasive TAVI may have serious complications that differ from those related to SAVR and include vascular injury, stroke, heart block, coronary obstruction, cardiac perforation, paravalvular leak, valve misplacement and PVE. Since only 13 years has elapsed from its first procedure implementation, long-term 
results regarding patient survival are still lacking. Thus, indication and appropriateness for TAVI should be cautiously individualized and patients must be advised on the possible complications of the technique.

Although during TAVI the heart chambers are not entirely opened and there is no requirement of extracorporeal circulation; the procedure is indicated in elderly or fragile patients. The latter factors may favour infection, including endocarditis. Additionally in most centres, such as ours, the procedure is done in the catheterization room without HEPA laminar flow [14]. Other factors that may favour infection after TAVI are the frequent paravalvular leak owing to imperfections in the anchor of the implanted valve, as well as the space between the bioprosthesis and the native valve cusp; which may serve as nidus for pathogen accumulation during transient bacteremia.

TAVI-PVE often present with subtle symptoms probably due to immunosenescence. The presence of fever is less frequent $(75 \%)$ than in cases of infective endocarditis in the general population $(90 \%)$ [16]. Non-specific symptoms such as asthenia or weight loss may be the only symptoms in up to one-fifth of the patients [16]. Also, the occurrence of a new regurgitant heart murmur, an important sign for the diagnosis of endocarditis, is less valuable in TAVI patients due to the high rate of residual paravalvular leak that persists following the procedure. Up to $20 \%$ of blood cultures are negative. These facts reduce the sensitivity of the Duke criteria leading to diagnostic delay and subsequent worse outcome $[17,18]$. An autopsy study of 13 patients deceased after TAVI found 3 cases of acute IE (23\%); none of them was identified while the patient was alive [19]. It is very important to have a low threshold for investigation of endocarditis to avoid under diagnosis.

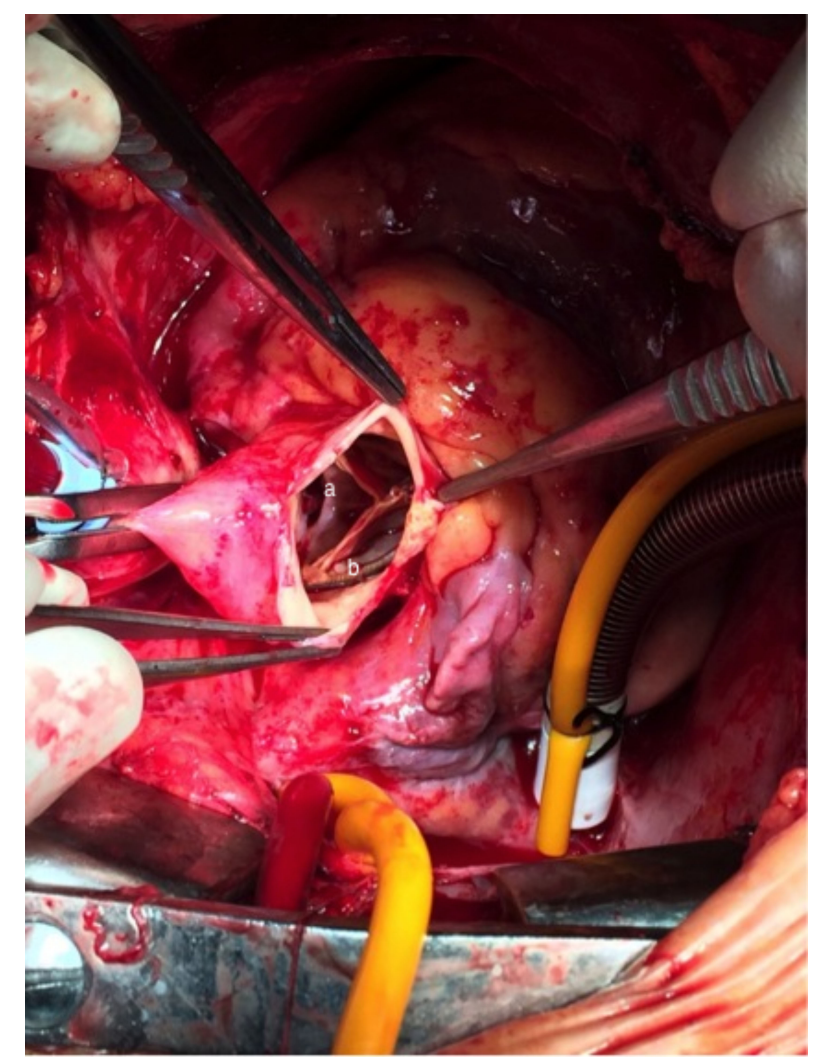

Fig. (3). TAVI prosthetic aortic valve endocarditis with leaflet perforation (a) and endocarditis vegetation (b).

TEE findings in TAVI-PVE also differ from those described in PVE, being periannular complications more frequent (39\% of TAVI-PVE compared with $30 \%$ of SAVR patients). Development of fistula has been described in $16 \%$ of TAVI-PVE patients versus 3\% in SAVR-PVE [13]. Additionally TEE may be difficult to interpret in patients with TAVI due to the shadowing effect and reflectance of the prosthetic material (metallic struts that encircle the valve leaflets). Detection of vegetations in the space between prosthetic and native aortic valve (usually calcified) is particularly difficult. Therefore meticulous examination at multiple views is advised. Eventually, other diagnostic modalities such as positron emission tomography-computed tomography could assist the diagnosis [20].

In the case we are reporting, the valve was well positioned with no leaks evidenced by 2 previous TEE performed 
one month before the diagnosis of PVE.

TAVI-PVE in-hospital mortality is $50 \%$ and 1 -year mortality is $66 \%$ [16]. These outcomes are striking when compared to in-hospital mortality for native valve endocarditis (15\%) and SAVR-PVE (40\%) [16]. Importantly, in TAVI-PVE poor results are related with conservative treatment highlighting the role open surgery in such patients despite their high surgical risk. In a German series 4 (26.7\%) of 15 patients with TAVI-PVE died; 3 of the 4 patients who died had strong indication for surgical therapy (abscesses, fistulas, pseudoaneurysms); nevertheless open-heart surgery was not done owing to the high risk calculated by scores [21].

\section{CONCLUSION}

PVE following TAVI is an emerging endovascular infection whose incidence seems to be slightly higher to that of PVE after SAVR. This could reflect the significant frailty of the patients as well as the learning curve in the technique. The particular features of TAVI candidates, as well as the characteristics of transcatheter valve prostheses, including a high amount of metal around the valve leaflets (stent frame), may alter the clinical manifestations, diagnosis and outcome of TAVI-PVE.

Health care acquisition and a distant primary source of infection are frequent in TAVI-PVE, as in the case we report. To the best of our knowledge this is the second report of TAVI-PVE due to P. aeruginosa, being the probable source an organ-space SSI. We cannot exclude the presence of PVE at the first manifestation of bacteremia accompanying the empyema, as TEE can have difficulties for detecting small vegetations in patients with TAVI because of the structure of the prosthetic valve and the calcification of the native valve that remains in situ.

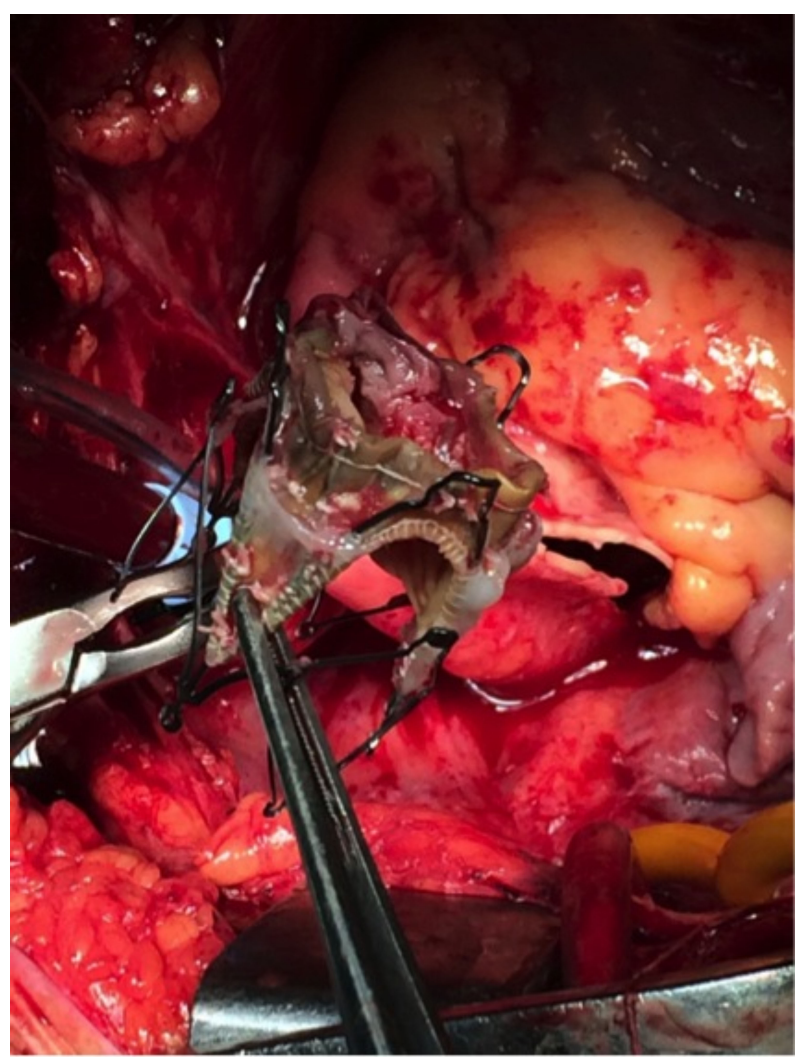

Fig. (4). Ectomized aortic TAVI valve with multiple vegetations.

The management of TAVI associated PVE is challenging especially when surgery is advised, as in the case of infection due to $P$. aeruginosa, because it occurs in patients previously considered inoperable. Nonetheless surgery seems to be associated with better outcome. Currently, the diagnosis, prevention and management of TAVI-PVE are based on clinical judgment guided by existing guidelines for PVE.

There are some pending questions that need to be resolved by further prospective studies. The safety of doing TAVI procedure in the catheterization room without HEPA laminar flow must be carefully assessed. Until more data are 
available it seems prudent to perform this procedure in hybrid surgery rooms with laminar flow. Probably preventive measures before TAVI procedure should be the same as with SAVR, including eradication of distant infectious sources (i.e. bacteriuria, cutaneous or dental infections), screening and treatment of nasal carriers of $S$. aureus, and timely antibiotic prophylaxis. Diagnostic criteria for TAVI-PVE must be specifically assessed by prospective clinical studies.

As experience grows it becomes clear that TAVI-PVE is an entity requiring specific considerations regarding diagnosis, prevention and treatment.

\section{CONFLICT OF INTEREST}

The authors confirm that this article content has no conflict of interest.

\section{ACKNOWLEDGEMENTS}

We thank Dr. David Wolf, Head of Echocardiography Department and Dr. Claudio Burgos, Head of Cardiovascular Surgery, for sharing images of the patient.

\section{REFERENCES}

[1] Nkomo VT, Gardin JM, Skelton TN, Gottdiener JS, Scott CG, Enriquez-Sarano M. Burden of valvular heart diseases: a population-based study. Lancet 2006; 368(9540): 1005-11.

[http://dx.doi.org/10.1016/S0140-6736(06)69208-8] [PMID: 16980116]

[2] Vahanian A, Alfieri OR, Al-Attar N, et al. Transcatheter valve implantation for patients with aortic stenosis: a position statement from the European Association of Cardio-Thoracic Surgery (EACTS) and the European Society of Cardiology (ESC), in collaboration with the European Association of Percutaneous Cardiovascular Interventions (EAPCI). Eur J Cardiothorac Surg 2008; $34(1)$ : 1-8. [http://dx.doi.org/10.1016/j.ejcts.2008.04.039] [PMID: 18502659]

[3] Kvidal P, Bergström R, Malm T, Ståhle E. Long-term follow-up of morbidity and mortality after aortic valve replacement with a mechanical valve prosthesis. Eur Heart J 2000; 21(13): 1099-111. [http://dx.doi.org/10.1053/euhj.2000.1862] [PMID: 10843828]

[4] Roques F, Nashef SA, Michel P, et al. Risk factors and outcome in European cardiac surgery: analysis of the EuroSCORE multinational database of 19030 patients. Eur J Cardiothorac Surg 1999; 15(6): 816-22. [http://dx.doi.org/10.1016/S1010-7940(99)00106-2] [PMID: 10431864]

[5] Shroyer AL, Coombs LP, Peterson ED, et al. The society of thoracic Surgeons: 30-day operative mortality and morbidity risk models. Ann Thorac Surg 2003; 75(6): 1856-64. [http://dx.doi.org/10.1016/S0003-4975(03)00179-6] [PMID: 12822628]

[6] Cribier A, Eltchaninoff H, Bash A, et al. Percutaneous transcatheter implantation of an aortic valve prosthesis for calcific aortic stenosis: first human case description. Circulation 2002; 106(24): 3006-8. [http://dx.doi.org/10.1161/01.CIR.0000047200.36165.B8] [PMID: 12473543]

[7] Martínez-Sellés M, Bouza E, Díez-Villanueva P, et al. Incidence and clinical impact of infective endocarditis after transcatheter aortic valve implantation. EuroIntervention 2015; 10(11): 20140826-07. [http://dx.doi.org/10.4244/EIJY15M02_05] [PMID: 25671426]

[8] Kodali SK, Williams MR, Smith CR, et al. Two-year outcomes after transcatheter or surgical aortic-valve replacement. N Engl J Med 2012; 366(18): 1686-95. [http://dx.doi.org/10.1056/NEJMoa1200384] [PMID: 22443479]

[9] Makkar RR, Fontana GP, Jilaihawi H, et al. Transcatheter aortic-valve replacement for inoperable severe aortic stenosis. N Engl J Med 2012; 366(18): 1696-704. [http://dx.doi.org/10.1056/NEJMoa1202277] [PMID: 22443478]

[10] Latib A, Naim C, De Bonis M, et al. TAVR-associated prosthetic valve infective endocarditis: results of a large, multicenter registry. J Am Coll Cardiol 2014; 64(20): 2176-8.

[http://dx.doi.org/10.1016/j.jacc.2014.09.021] [PMID: 25457406]

[11] Aung T, Poon K, Horvath R, Coulter C, Walters DL. A case series of medically managed infective endocarditis after transcatheter aortic valve replacement. Scand J Infect Dis 2013; 45(6): 489-93. [http://dx.doi.org/10.3109/00365548.2012.754105] [PMID: 23286779]

[12] López J, Revilla A, Vilacosta I, et al. Definition, clinical profile, microbiological spectrum, and prognostic factors of early-onset prosthetic valve endocarditis. Eur Heart J 2007; 28(6): 760-5. [http://dx.doi.org/10.1093/eurheartj/ehl486] [PMID: 17255216]

[13] Pericas JM, Llopis J, Cervera C, et al. Infective endocarditis in patients with an implanted transcatheter aortic valve: Clinical characteristics and outcome of a new entity. J Infect 2015; 70(6): 565-76. [http://dx.doi.org/10.1016/j.jinf.2014.12.013] [PMID: 25561168] 
[14] Onsea K, Agostoni P, Voskuil M, Samim M, Stella PR. Infective complications after transcatheter aortic valve implantation: results from a single centre. Neth Heart J 2012; 20(9): 360-4. [http://dx.doi.org/10.1007/s12471-012-0303-9] [PMID: 22890618]

[15] Remmelts HH, Meine M, Loh P, et al. Infection after ICD implantation: operating room versus cardiac catheterisation laboratory. Neth Heart J 2009; 17(3): 95-100. [http://dx.doi.org/10.1007/BF03086226] [PMID: 19325900]

[16] Amat-Santos IJ, Ribeiro HB, Urena M, et al. Prosthetic valve endocarditis after transcatheter valve replacement: a systematic review. JACC Cardiovasc Interv 2015; 8(2): 334-46. [http://dx.doi.org/10.1016/j.jcin.2014.09.013] [PMID: 25700757]

[17] Eisen A, Shapira Y, Sagie A, Kornowski R. Infective endocarditis in the transcatheter aortic valve replacement era: comprehensive review of a rare complication. Clin Cardiol 2012; 35(11): E1-5. [http://dx.doi.org/10.1002/clc.22052] [PMID: 22990884]

[18] Amat-Santos IJ, Messika-Zeitoun D, Eltchaninoff H, et al. Infective endocarditis after transcatheter aortic valve implantation: results from a large multicenter registry. Circulation 2015; 131(18): 1566-74. [http://dx.doi.org/10.1161/CIRCULATIONAHA.114.014089] [PMID: 25753535]

[19] Loeser H, Wittersheim M, Puetz K, Friemann J, Buettner R, Fries JW. Potential complications of transcatheter aortic valve implantation (TAVI)-an autopsy perspective. Cardiovasc Pathol 2013; 22(5): 319-23. [http://dx.doi.org/10.1016/j.carpath.2013.01.006] [PMID: 23395535]

[20] Bartoletti M, Tumietto F, Fasulo G, et al. Combined computed tomography and fluorodeoxyglucose positron emission tomography in the diagnosis of prosthetic valve endocarditis: a case series. BMC Res Notes 2014; $7: 32$. [http://dx.doi.org/10.1186/1756-0500-7-32] [PMID: 24418206]

[21] Puls M, Eiffert H, Hünlich M, et al. Prosthetic valve endocarditis after transcatheter aortic valve implantation: the incidence in a single-centre cohort and reflections on clinical, echocardiographic and prognostic features. EuroIntervention 2013; 8(12): 1407-18. [http://dx.doi.org/10.4244/EIJV8I12A214] [PMID: 23015071]

Received: August 22, $2015 \quad$ Revised: September 20,2015

Accepted: October 22, 2015

(C) Dapás et al.; Licensee Bentham Open.

This is an open access article licensed under the terms of the Creative Commons Attribution-Non-Commercial 4.0 International Public License (CC BY-NC 4.0) (https://creativecommons.org/licenses/by-nc/4.0/legalcode), which permits unrestricted, non-commercial use, distribution and reproduction in any medium, provided the work is properly cited. 